\title{
PROCESSOS COGNITIVOS BÁSICOS E EDUCAÇÃO
}

\author{
Basic Cognitive Processes and Education
}

Gerson Américo Janczura1

\section{Resumo}

Este artigo focaliza a relação entre os processos cognitivos básicos e a educação, objetivando apresentar algumas contribuições da pesquisa básica. Relata-se como as pesquisas sobre a memória, a atenção, a resolução de problemas e metacognição podem orientar educadores a melhorar o desempenho dos alunos. São discutidos, ainda, alguns obstáculos que têm dificultado a aplicação do conhecimento gerado pela pesquisa como a falta de comunicação entre pesquisadores e educadores, a necessidade da educação ser baseada cientificamente, a falta de treinamento dos professores e a necessidade de generalização do conhecimento para diferentes contextos, entre outros. Sugerem-se, por fim, altemativas para superar as dificuldades apontadas.

Palavras-chave: Processos cognitivos básicos; Educação; Memória; Atenção; Resolução de problemas; Metacognição.

\section{Abstract}

This paper addresses the relation between basic cognitive processesand education. It aims at presenting some contributions from basic research to education. It is illustrated how research on memory, attention, problem solving and metacognition may help educators in improving students' performance. The paper also points out some obstacles in applying the knowledge produced by basic research to learning contexts such as poor communication between researchers and teachers, an education not scientifically grounded, the lack of training programs for teachers, and the generalization power of knowledge to different contexts.

Keywords: Basic cognitive processes; Education; Memory; Attention; Problem solving; Metacognition.

1 Psicólogo. Mestre em Psicologia pela Universidade de Brasília. Ph.D. pela University of South Florida/USA. Coordenador do Laboratório de Processos Cognitivos/UnB.

Endereço para contato: Universidade de Brasília - Câmpus Darcy Ribeiro, Instituto de Psicologia/PPB, Brasília -DF. CEP: 70910900.

E-mail: janczura@unb.br

Psicol. Argum., Curitiba, v. 24, n. 46 p. 39-44, jul./set. 2006. 
A Educação, como uma disciplina da Ciência Cognitiva, tem como aspecto central investigar a aprendizagem mediante pesquisas experimentais, teóricas e aplicadas. Usualmente adotase uma abordagem mais processual dirigida à aquisição, desenvolvimento, acesso, utilização e representação do conhecimento. Trata-se de uma área que busca apoio multidisciplinar em outras disciplinas como a lingüística, a neurociência, a antropologia e a psicologia cognitiva (Ranney \& Shimoda, 1999). As pesquisas sobre processos educacionais relacionados à aprendizagem humana incluem vários tópicos como, por exemplo, desenvolvimento da perícia, aquisição de habilidades, metacognição, esquemas, modelos mentais, cognição espacial, raciocínio dedutivo e indutivo, resolução de problemas, atenção, representação do conhecimento e memória. Este artigo tem por objetivo apresentar algumas das questões envolvidas no binômio "processos cognitivos básicos e educação", no que diz respeito à relação entre estas disciplinas, as dificuldades desta relação, algumas contribuições e desafios à pesquisa básica sobre os processos cognitivos no contexto educacional. A motivação da discussão é promover a aproximação entre a pesquisa básica em cognição e sua aplicação no campo da educação, assim como ilustrar algumas contribuições dos processos cognitivos básicos à aprendizagem.

Várias são as contribuições da pesquisa sobre os processos cognitivos básicos para a educação. Ilustrando, o conhecimento sobre o funcionamento do processo básico da memória pode orientar o educador a como melhorar o desempenho de seus alunos na memorização, a longo prazo, de conteúdos ensinados em sala de aula; a pesquisa sobre raciocínio humano aponta como melhorar a resolução de problemas que envolvam argumentos naturalísticos; fatores envolvidos na resolução de problemas podem ser ensinados aos educando de forma a evitar as várias armadilhas que desfavorecem a solução de problemas e o pensamento criativo.

Outra contribuição é o desenvolvimento de ambientes computacionais que beneficiam a aprendizagem nas áreas de ciência, matemática, compreensão da leitura e da escrita. Programas de computadores podem ser utilizados para auxiliar na aprendizagem de conceitos centrais de diferentes áreas do conhecimento.
Algumas contribuições mais específicas também podem ser apontadas: os resultados das pesquisas sobre o funcionamento da atenção contribuem para o desenvolvimento de materiais de leitura que favorecem a focalização da atenção reduzindo as distrações dos alunos.

Os resultados das pesquisas sobre a memória humana podem orientar os educadores a escolherem os materiais para os estudantes e a dosar a quantidade de informação de forma a beneficiar a armazenagem e recuperação do conhecimento a longo-prazo. Além disso, professores podem ser orientados a organizar os conteúdos programáticos de forma significativa de maneira a aumentar a probabilidade da lembrança posterior das informações. Os estudos sobre o processo básico da memória mostram que algumas maneiras de armazenar as informações por longos períodos de tempo são mais produtivas do que outras. Por exemplo, a aprendizagem significativa é mais efetiva do que a aprendizagem por repetição, ou seja, associar as novas informações às informações já armazenadas na memória está associada a uma maior probabilidade de reter e lembrar a informação. Pesquisas têm mostrado que livros escolares apresentam listas de fatos que exibem pouca relação entre si e não consideram o conhecimento adquirido pelos estudantes (Beck \& McKeown, 1988; Chambliss, Calfee \& Wong, 1990). Nesse mesmo sentido, a elaboração e a organização interna da informação também facilitam a armazenagem e a recuperação do conhecimento.

$\mathrm{Na}$ área da categorização, as pesquisas sobre a aquisição, a representação e a utilização de conceitos produziram várias implicações educacionais. Mostrou-se, por exemplo, que a aprendizagem de conceito s é facilitada quando são apresentados vários exemplos de categorias e também quando são apresentados itens que não são exemplos de categorias (Clark, 1971; Tennyson \& Cochiarella, 1986; Freiburgs \& Tulving, 1961). Esta área também tem sugerido formas de avaliar a aprendizagem mostrando que a compreensão conceitual pode ser verificada solicitando ao estudante que classifique novos exemplos em categorias, ou que produzam exemplos de categorias conceituais e aplicações de um conceito (Kinnick 1990; Ellis \& Hunt, 1983; Watts \& Anderson, 1971).

Os pesquisadores na área da metacognição têm sugerido práticas que contribuem para 0 desenvolvimento do conhecimento metacognitivo 
e de habilidades como conhecer quais estratégias de aprendizagem são mais efetivas, monitorar 0 próprio conhecimento, conhecer estratégias efetivas para a evocação da informação memorizada, como usar efetivamente estratégias de aprendizagem (Van Rossum \& Schenk, 1984; Dole, Duffy, Roehler \& Person, 1991; Benton, Kiewra, Whitfill \& Dennison, 1993; Baker, 1989; Nist, Simpson, Olejnik \& Mealey, 1991; Pressley, Harris \& Marks, 1992).

Entretanto, as contribuições da pesquisa básica têm encontrado obstáculos que viabilizem a aplicação de seus resultados à educação. Verifica-se, primeiramente, falta de comunicação entre os pesquisadores dos processos básicos e educadores. A reduzida interação entre estes grupos é prejudicial a ambos. Por um lado, os cientistas não se beneficiam das observações oriundas do contexto educacional na produção do conhecimento (p.ex., geração e testagem de hipóteses, avaliação de teorias e modelos, modificações na metodologia de investigação, avaliação da validade ecológica de seus resultados/conhecimento). Por outro lado, a educação não se beneficia do conhecimento sobre os processos básicos, ou a sua aplicação não é apropriada.

A comunidade científica deveria influenciar as políticas educacionais. As diretrizes e ações do sistema educacional deveriam ter um apoio sólido no conhecimento científico, em vez de estarem motivadas politicamente. Técnicas educacionais deveriam estar fundamentadas em evidências científicas sólidas. Esta proposta implica em uma educação que

envolva a aplicação de procedimentos rigorosos, sistemáticos e objetivos a fim de obter conhecimento válido e confiável relacionados à educação; envolva pesquisa que utilize métodos empíricos e sistemáticos que gerem dados válidos e confiáveis para diferentes educadores, participantes, medidas, observações e pesquisadores; seja avaliada através de delineamentos experimentais ou quaseexperimentais nos quais indivíduos, instituições, programas ou atividades sejam designados randomicamente a diferentes condições, adotando-se controles apropriados ou, ainda, que outros delineamentos sejam propostos contanto que incluam controles intra e intercondições; garanta que estudos experimentais sejam apresentados com clareza e sufici- ência de detalhes que permita a sua replicação, e que seus resultados tenham sido aceitos através de uma revisão científica rigorosa e objetiva realizada por pares associados a periódicos científicos ou executada por um grupo de especialistas (Murray, 2002, p. 53).

Desenvolver uma educação, baseada cientificamente, não é um objetivo que poderá ser alcançado somente com as contribuições da pesquisa sobre os processos cognitivos básicos envolvidos na educação. Considerando a multiplicidade e complexidade do campo educacional, é condição necessária integrar o conhecimento sobre os processos cognitivos a outras áreas da psicologia e disciplinas relacionadas como a ciência da computação, lingüística, psicologia do desenvolvimento, psicologia social. Isto significa focalizar de maneira multidisciplinar a pesquisa e a aplicação do conhecimento sobre a aprendizagem humana. A psicologia social pode, por exemplo, examinar como o ambiente social influencia o desempenho escolar, a antropologia pode contribuir sobre como a cultura influencia a aprendizagem, a psicobiologia pode contribuir sobre as bases neurológicas da aprendizagem, e os pesquisadores sobre memória podem contribuir sobre técnicas que maximizem a manutenção da informação a longo prazo. Esta abordagem sugere a criação de centros de pesquisa interdisciplinares sobre cognição, instrução e treinamento de professores que congregaria diferentes perspectivas e disciplinas para tratarem das questões complexas da educação e aprendizagem (Azar, 2002).

A integração de conhecimentos de diferentes disciplinas é uma estratégia mais apropriada para enfrentar os desafios da educação considerando a multideterminação dos produtos e processos da educação, a complexidade das escolas e do próprio processo educacional. Tradicionalmente, a pesquisa em laboratório sacrifica a complexidade de um fenômeno em prol de considerações metodológicas. Isto implica na definição do objeto de análise. No contexto da educação qual seria o objeto?Algumas alternativas são: tarefas, indivíduos, estímulos, escola, situações, os processos básicos, a escola, a aprendizagem. No caso do foco investigativo serem os processos cognitivos é relevante avaliar se o desempenho em tarefas laboratoriais corresponderia a uma amostra representativa do desempenho fora do contexto experimen- 
tal. Simon (1976) propôs um paradigma experimental que pode ser utilizado para verificar a validade ecológica dos achados experimentais:

identificamos duas tarefas que possuam a mesma estrutura formal (por exemplo, ambas são tarefas de julgamento multidimensional), sendo que uma foi selecionada de uma situação social e a outra não o foi. Se as tarefas partilham processos comuns, então nós deveríamos ser capazes de produzir, no ambiente de cada tarefa, fenômenos que evidenciam a atuação dos mesmos mecanismos cognitivos (p. 258; tradução livre).

A proposta de uma educação apoiada cientificamente será insuficiente se os professores não forem preparados para desenvolverem suas atividades baseadas no conhecimento científicas. Isto significa treiná-los para fazer a aplicação dos princípios psicológicos básicos voltados à prática (Murray, 2002). Além disso, é necessário que os psicólogos apresentem suas pesquisas de maneira que os professores possam acessá-las e estender para o contexto em que atuam, e isto implica na utilização de uma linguagem adequada. Várias são as alternativas que viabilizariam a preparação dos professores, incluindo-se cursos de atualização ou especialização, inserção de disciplinas da psicologia sobre processos básicos no currículo de formação dos educadores, melhorar a comunicação entre pesquisadores e educadores, participação de cientistas na formulação de políticas educacionais nas agências governamentais, divulgação do conhecimento científico, formação de centros de pesquisa multidisciplinares, entre outros.

Uma das tarefas-meta que deve ser permanentemente executada no binômio "processos básicos-educação" é a transferência, generalização do conhecimento para diferentes contextos. Esta meta pode se tornar mais provável e rápida na medida em que as nossas teorias cognitivas exibirem validade ecológica, expressão cunhado por Neisser (1976) para se referir à busca de teorias que descrevam a utilização do conhecimento pelos indivíduos em situações reais, cotidianas, culturalmente significativas. Além disso, a contribuição da pesquisa sobre processos básicos na educação dependerá de sua aplicabilidade e replicação nas diversas situações escolares. Esta exigência é severa considerando as diferenças entre instituições e situações como métodos de ensino, fai- xas etárias, sistemas de valores, níveis de habilidade, conteúdo curricular, entre outros. É importante relembrar, como foi apontado anteriormente, que as escolas são ambientes muito complexos e multifacetados e que a transferência do conhecimento produzido em laboratório para o cotidiano escolar não deve ser pressuposto (Sternberg \& Lyon, 2002).

A satisfação da tarefa-meta, acima mencionada, depende da disponibilização do conhecimento científico não somente à comunidade científica, como usualmente ocorre pelos meios tradicionais de divulgação como periódicos, eventos científicos e sociedades, entre outros, mas, também, aos educadores das diversas áreas do conhecimento. Estes canais formais de comunicação científica têm as seguintes características, segundo Mardero Arellano, Melo, Barboza, Mendes, Café e Lage (2002), são morosos na produção e distribuição do conhecimento, não promovem a interação entre os pares, têm custo elevado e desigualdade de acesso aos interessados. Por outro lado, ainda são poucos os pesquisadores que utilizam recursos eletrônicos que viabilizam o acesso aberto à informação científica como é o caso dos Arquivos Abertos (AA). Marcondes, Kuramoto, Toutain e Sayão (2005) definem AA como arquivos eletrônicos que contêm informação científica ou cultural, geralmente de acesso livre, dotados de dispositivos de autopublicação e intercâmbio de metadados, isto é, elementos de descrição, definição e avaliação de recursos informacionais armazenados em sistemas computadorizados. Os arquivos abertos são muito interessantes por várias razões, de acordo com Harnad (1999): exibem maior rapidez na disseminação da literatura científica publicada nestes repositórios, aumentam a visibilidade dos trabalhos publicados, não têm custos de distribuição e reprodução nem negociações específicas, produzem maior impacto em função de estarem disponíveis para acesso livre promovendo, assim, maior visibilidade e probabilidade de serem citados e utilizados.

No Brasil verificam-se, no momento, 26 AA (Institution Archives Registry, 2005), sendo que seis destes disponibilizam informações úteis à psicologia ou educação que são: Scielo (http:// www.scielosp.org), ETD - Educação Temática Digital (http://143.106.58.55), ETD - Educação Temática Digital (http://143.106.58.55), Biblioteca Digital de Teses e Dissertações (http:// www.teses.usp.br), Universidade Federal do Paraná: Biblioteca Digital de Teses e Dissertações (http:/ 
/dspace.c3sl.ufpr.br), e Pró-Reitoria de Pesquisas e Pós-Graduação (http://www.prppg.ufpr.br/ coord stricto/revista/revistas.html). Consultando o cadastro de periódicos que utiliza o sistema eletrônico de editoração de revistas SEER/IBICT (2005) e a base de dados de textos completos SCIELO/ BIREME (2005), verifica-se que o número de periódicos de acesso livre, atualmente disponíveis no Brasil, é nove que são: Estudos de Psicologia (UFRN/Natal), Psicologia \& Sociedade (Associação Brasileira de Psicologia Social), Psicologia USP (Universidade de São Paulo), Psicologia em Estudo (Universidade Estadual de Maringá), Psicologia: Reflexão e Crítica (UFRGS), Psicologia: Teoria e Pesquisa (Unb), Interação em Psicologia (UFPR), Psicologia e Sociedade (Associação Brasileira de Psicologia Social), Psicologia: Teoria e Prática (Universidade Presbiteriana Mackenzie).

Finalmente, é crítico lembrar que a produção de conhecimento científico sobre o binômio "processos básicos-educação" depende de investimento financeiro na pesquisa e na formação de pesquisadores de alto nível. O montante de investimento que o Brasil destina a esta área é muito pequeno. Uma análise das séries históricas do Conselho Nacional de Desenvolvimento Científico e Tecnológico (CNPq, 2005a) evidencia que, em 2004, o investimento na grande área de Humanidades, na qual estão inseridas a Psicologia e a Educação, é equivalente à metade dos recursos financeiros alocados às demais grandes áreas que são Ciências da Natureza e Ciências da Vida, correspondendo a $20 \%$ do total de recursos da agência de fomento. Esta proporção se mantém quando são avaliados os recursos destinados às três áreas no período de 1999 a 2004. No ordenamento das áreas de conhecimento, com base no total de investimentos realizados em bolsas de estudo e fomento à pesquisa (CNPq, 2005b), constata-se que a Psicologia ocupa a 19a. Posição e a Educação a 7ạ. Posição entre setenta e seis áreas, sendo as cinco primeiras posições ocupadas por Agronomia, Química, Física, Ciência da Computação e Engenharia Elétrica, respectivamente. A participação percentual média da Psicologia no período de 1999 a 2004 é de 2,14\% e da Educação é 2,80\%. É preocupante a verificação de uma diminuição progressiva nos investimentos destinados à Psicologia que eram de 2,36\% em 1999 e caíram para 1,85 em 2004.

\section{Comentários Finais}

Este depoimento propôs-se a discorrer como o conhecimento científico sobre os processos cognitivos básicos pode influenciar e contribuir para o aprimoramento da educação, tanto no que se relaciona às práticas educacionais, ao desempenho do professor e aluno, aos materiais educacionais e ao contexto da aprendizagem de maneira geral. Apesar de muito conhecimento ter sido produzido pelos pesquisadores da área, verifica-se, ainda, que pouco conhecimento tem sido utilizado no ambiente escolar. Várias foram as razões elencadas: falta de comunicação entre pesquisadores e educadores, falta de treinamento dos educadores, necessidade da criação de centros que abordem a educação de maneira multidisciplinar, necessidade de disponibilizar o conhecimento científico de maneira mais efetiva para os educadores, entre outros. Argumentou-se, ainda, que o sucesso do binômio processos básicos-educação depende do desenvolvimento de políticas educacionais baseadas cientificamente e do investimento financeiro significativo em pesquisa na área de Ciências Humanas.

\section{Referências}

Azar, B. (2002). The science of learning. Monitor on Psychology, 33(8), 60-62.

Baker, L. (1989). Metacognition, comprehension monitoring, and the adult reader. Educational Psychology Review, 1, 3-38.

Beck, I. L. \& McKeown, M.G. (1988). Toward meaningful accounts in history texts for young learners. Educational Researcher, 47(6), 31-39

Benton, S. L., Kiewra, K. A., Whitfill, J. M., \& Dennison, R. (1993). Encoding and external-storage effects on writing processes. Journal of Educational Psychology, 85, 267-280.

Chambliss, M. J., Calfee, R. C., \& Wong, I. (1990). Structure and context in science textbooks: Where is the design? Paper presented at the annual meeting of the American Educational Research Association Boston, MA. 
Clark, D. C. (1971). Teaching concepts in classroom: A set of teaching prescriptions derived from experimental research. Journal of Educational Research, 62, 253-278.

CNPq. (2005a). Séries históricas. Recuperado em 6 de julho de 2005:

http:// ww w.cnpq.br/ servicos/ estatisticas/ historicas.htm.

CNPq (2005b). Séries históricas. Recuperado em 6 de julho de 2005: http://ftp.cnpq.br/pub/doc/ aei/T20-AreaTot_9904_site.pdf.

Dole, J. A; Duffy, G. G.; Roehler, L. R. \& Pearson, P. D. (1991). Moving from the old to the new: Research on reading comprehension instruction. Review on Educational Research, 61, 239-264.

Ellis, H. C. \& Hunt, R. R. (1983). Fundamentals of human memory and cognition (3 ed.). Dubuque, I. A: Wm C. Brown.

Freiburgs, V. \& Tulving, E. (1961). The effect of practice on utilization of information from positive and negative instances in concept identification. Canadian Journal of Psychology, 15, 101-106.

Harnad, S. (1999). Free at last: The future of peerreviewed journals. D-Lib Magazine, 5(12). Recuperado em 5 de Janeiro de 2005: http:// www.dlib.org/dlib/december99/12harnad.html.

Institution Archives Registry (2005). Recuperado em 18 de julho de 2005: http://archives.eprints.org/ index.php?action $=$ home\&country $=$ br..

Kinnick, V. (1990). The effect of concept teaching in preparing nursing students for clinical practice. Journal of Nursing Education, 29, 362-366.

Marcondes, C. H.; Kuramoto, H.; Toutain, L B. \& Sayão, L (2005). Bibliotecas Digitais: Saberes e Práticas. Salvador: Brasília: UFBA: IBICT.

Márdero Arellano, M. A., Melo, B. M., Barboza, E. M. F., Mendes, E., Café, L., \& Lage, M. B. (2002). Arquivos Abertos: inovação para a comunicação científica na Rede. In: Anais, 25 Congresso Brasileiro de Ciências da Comunicação, 25 e 12. ENDOCOM, 2002, Salvador.

Murray, B. (2002). Wanted: politics-free, sciencebased education. Monitor on Psychology, 33(8), 52-54.
Neisser, U. (1876). Cognition and reality: Principles and implications of cognitive psychology. San Francisco: Freeman.

Nist, S. L; Simpson, M. L; Olejnik, S., \& Mealey, D. L. (1991). The relation between self-select study processes and test perfiormance. American Educational Research Journal, 28, 849-874.

Pressley, M.; Harris, K. R. \& Marks, M. B. (1992). But good strategy instructors are constructivists! Educational Psychology Review, 4, 3-31.

Ranney, M. \& Shimoda, T. (1999). In R. A Wilson e F. C. Keil (Eds.), The MIT encyclopedia of Cognitive Science, Cambridge: MIT Press.

SCIELO/BIREME (2005). Recuperado em 7 de julho de 2005: http://www.scielo.org.

SEER/IBICT (2005). Recuperado em 7 de julho de 2005: http://www.ibict.br/ seer.

Simon, H. A. (1976). Discussion: Cognition and social behavior. In J. S. Carroll and J. W. Payne, Eds., Cognition and Social Behavior. Hillsdale, N. J: Erlbaum.

Sternberg, R. J., \& Lyon, G. R. (2002). Making a difference to education: Will psychology pass up the chance? Monitor on Psychology, 33(7), 7677.

Tennyson, R. D., \& Cochiarella, M. J. (1986). An empirically based instructional design theory for teaching concepts. Review of Educational Research, 56, 40-71.

Van Rossum, B., \& Schenk, S. M. (1984). The relationship between learning conception, study strategy and learning outocome. British Journal of Educational Psychology, 54, 73-83.

Watts, G. H., \& Anderson, R. C. (1971). Effects of three types of inserted questions on learning from prose. Journal of Educational Psychology, 62, 387-394.

Recebido em/received in: 01/02/2005 Aprovado em/approved in: 21/03/2006 